\title{
Crop changes from the XVI century to the present in a hill/mountain area of eastern Liguria (Italy) Rodolfo Gentili*1, Elio Gentili $^{2}$ and Sergio Sgorbati ${ }^{1}$
}

Address: ${ }^{1}$ Dipartimento di Scienze dell'Ambiente e del Territorio, Università degli Studi di Milano-Bicocca, Piazza della Scienza n. 1, I-20126, Milano, Italy and 2Biblioteca Niccolò V, Archivi Vescovili Lunensi, Via Mascardi n. 93, I-19038 - Sarzana (SP), Italy

Email: Rodolfo Gentili* - rodolfo.gentili@unimib.it; Elio Gentili - elio.gentili@libero.it; Sergio Sgorbati - sergio.sgorbati@unimib.it

* Corresponding author

Published: II April 2009

Journal of Ethnobiology and Ethnomedicine 2009, 5:9 doi:10.1186/1746-4269-5-9

This article is available from: http://www.ethnobiomed.com/content/5/I/9

(C) 2009 Gentili et al; licensee BioMed Central Ltd.

This is an Open Access article distributed under the terms of the Creative Commons Attribution License (http://creativecommons.org/licenses/by/2.0), which permits unrestricted use, distribution, and reproduction in any medium, provided the original work is properly cited.
Received: 24 October 2008

Accepted: II April 2009

\begin{abstract}
Background: Chronological information on the composition and structure of agrocenoses and detailed features of land cover referring to specific areas are uncommon in ethnobotanical studies, especially for periods before the XIX century. The aim of this study was to analyse the type of crop or the characteristics of soil cover from the XVI century to the present.
\end{abstract}

Methods: This diachronic analysis was accomplished through archival research on the inventories of the Parish of St. Mary and those of the Municipality of Pignone and from recent surveys conducted in an area of eastern Liguria (Italy).

Results: Archival data revealed that in study area the primary means of subsistence during the last five centuries, until the first half of the XX century, was chestnuts. In the XVIII and XIX centuries, crop diversification strongly increased in comparison with previous and subsequent periods. In more recent times, the abandonment of agricultural practices has favoured the re-colonisation of mixed woodland or cluster-pine woodland.

Conclusion: Ancient documents in the ecclesiastic or municipal inventories can be a very useful tool for enhancing the knowledge of agricultural practice, as well as of subsistence methods favoured by local populations during a particular time and for reconstructing land use change over time.

\section{Background}

One frequent problem in assessing the causes and degree of changes in land use and crop diversity through time has been the scarcity of historical data for documenting such changes [1]. Unfortunately, archival documentation has either not been preserved or is difficult to find, especially pertaining to periods prior to the XIX century.

The Council of Trent (1545-1563), introduced the bishops' requirement for periodic inspection of the invento- ries in each parish, convent or other ecclesiastic institution (including cultivated fields, forests, mills, etc.), encouraging their compilation [2]. Such documents, used rarely in ethnobotanical investigation up to now, can be a very important source of chronological information on the composition and/or structure of agrocenoses and detailed features of land cover when specific for certain areas or even toponyms [3]. Therefore, examination of the ecclesiastic possession inventories can enhance the knowledge of agricultural and livestock practice and subsistence 
methods of local populations during this time. In addition, other historical sources, such as cadastral maps, tax registers and manorial accounts, can give useful information about agricultural practices [4]. Furthermore, indirect data regarding the natural environment (i.e. land cover) can be extrapolated from ancient archival documents.

It is well known that in past centuries subsistence agriculture was the main source of food for European people. Everywhere, cereals were the most important crop. Locally, cereals were partially replaced by other farm products, such as potatoes, legumes, olives, and chestnuts [5].

During the second half of the XX century, in many hill/ mountain regions of Europe (especially those close to the Mediterranean Sea) abandonment of mall-scale or household agriculture increased [6]. This abandonment occurred as humans began to systematically use extensive farming techniques, mostly in planned areas, and under the impacts of industrial/economic development [7].

Time-series investigations of changes in crop variety, even on a small scale, might uncover insights with broader regional implications [8].

In this article, we attempt to reconstruct changes in the type of crops and the characteristics of soil cover during the time from the XVI century to the present through archival and field research conducted on the inventories of the Parish of St. Mary and the Pignone territory in eastern Liguria.

\section{Methods \\ Study area}

The Municipality of Pignone lies in eastern Liguria, in Val di Vara, at the border with the National Park of the Cinque Terre, less than 10 kilometres from the Ligurian Sea (Figure 1); it rises $189 \mathrm{~m}$ above sea level. The highest peak in the area reaches about 600 meters. In the study area, a Site of Community Importance (SCI), related to a karstic zone, is present.

From a historical point of view, the origin of Pignone village is very ancient, as indicated by archaeological findings from the Bronze Age on Mount Castellaro [9]. The same name, "Castellaro", indicates the presence of Ligurian population settlements. In the Middle Ages, this area was the domain of the Malaspina dynasty, the bishops of Luni, the Fieschi dynasty and the Genova Republic [10].

The climate of the study area is sub-Mediterranean and characterised by warm summers and temperate winters; precipitation is mostly concentrated in spring and autumn.

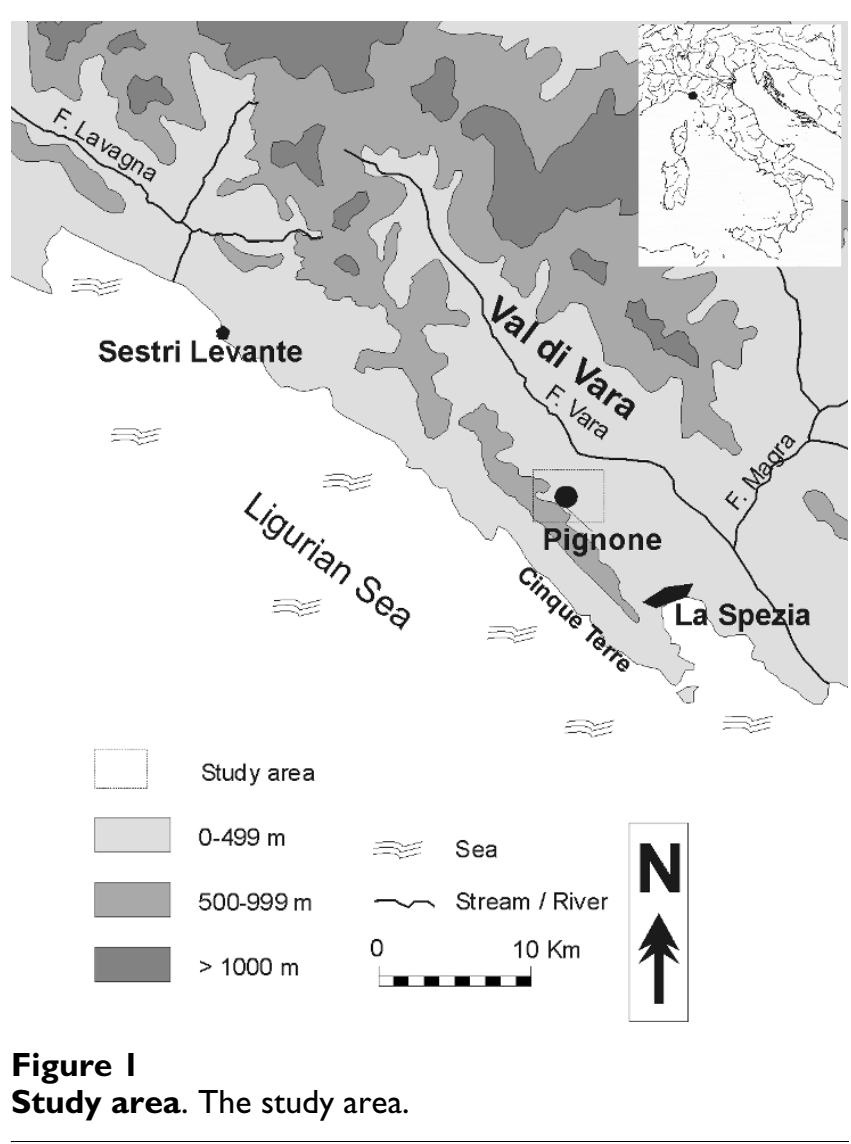

From a phytogeographic point of view, the study area is located in the Italo-Thyrrenian province, within the Mediterranean region [11].

The natural vegetation present in the Pignone territory mainly consists of holm-oak (Quercus ilex L.) mixed woodland with a high cover of downy-oak (Quercus pubescens Willd.). In a wide portion of the territory, cluster-pine (Pinus pinaster Aiton) anthropogenic and semi-natural (favoured by fire) woodlands are present. On fresh slopes, a mixed woodland dominated by European hop-hornbeam (Ostrya carpinifolia Scop.), manna-ash (Fraxinus ornus L.) and downy-oak is present. Locally, the vegetation is characterised by anthropogenic woods of chestnut (Castanea sativa Mill.). Black-alder (Alnus glutinosa (L.) Gaertn.) woodlands grow at the bottom of valleys, along with willow (Salix spp.) formations.

\section{Archival data and field survey}

In this study, five time periods since the XVI century are compared according to their crop or land use in the Pignone territory in the following years: 1588, 1663, $1763,1828,1878,1933$, and 2008 (Table 1, Table 2). The types of crops have been obtained through documentary investigation of all archival folders existing relating to the Parish of St. Mary in Pignone and to the Municipality of 
Table I: Inventories (part A)

\begin{tabular}{|c|c|c|c|c|c|c|c|}
\hline \multicolumn{8}{|c|}{ Type of crop } \\
\hline Toponym & 1588 & 1663 & 1763 & 1828 & 1878 & 1933 & 2008 \\
\hline A piè di Cravadora & $\mathrm{Cg}$ & $\mathrm{Cg}$ & $\mathrm{Cg}$ & $\mathrm{Cg}, \mathrm{W}$ & $x$ & $x$ & $\mathrm{Cg}, \mathrm{W}$ \\
\hline Alla Focina & $M$ & $x$ & $x$ & $x$ & $x$ & $x$ & W \\
\hline Alla Presa & $x$ & $\mathrm{Hf}$ & $x$ & $\mathrm{x}$ & $x$ & $\mathrm{Cg}$ & $x$ \\
\hline Aretta & $M$ & $\mathrm{Hf}$ & $\mathrm{M}, \mathrm{Ar}$ & $\mathrm{Al}, \mathrm{Vy}, \mathrm{Ar}$ & $x$ & $x$ & W \\
\hline Borela & $x$ & $x$ & $x$ & $\mathrm{Cg}$ & $x$ & W & $B, W$ \\
\hline Calcinarla I & $M$ & $\mathrm{Hf}$ & $x$ & $\mathrm{Al}, \mathrm{Vy}, \mathrm{Ar}$ & $\mathrm{Al}, \mathrm{Vy}, \mathrm{Ar}$ & $x$ & B \\
\hline Calcinarla 2 & $\mathrm{M}, \mathrm{Hf}$ & $\mathrm{Hf}$ & $\mathrm{M}, \mathrm{Ar}$ & $\mathrm{Al}, \mathrm{Vy}, \mathrm{Ar}$ & $\mathrm{Al}, \mathrm{Vy}$ & $\mathrm{Al}, \mathrm{Vy}$ & $\mathrm{B}, \mathrm{Al}$ \\
\hline Campedio* & $M$ & Og & $x$ & $x$ & $x$ & $x$ & $x$ \\
\hline Campuscasco I & $x$ & $\mathrm{Cg}$ & $x$ & $x$ & $\mathrm{Cg}, \mathrm{W}$ & $\mathrm{Cg}, \mathrm{W}$ & W \\
\hline Canavaro* & $x$ & $\mathrm{Hf}$ & $\mathrm{Hf}$ & $\mathrm{Al}, \mathrm{Vy}, \mathrm{Ar}$ & $x$ & $\mathrm{Vy}, \mathrm{Al}$ & $x$ \\
\hline Carpena & $\mathrm{Cg}$ & $x$ & $x$ & $\mathrm{x}$ & $x$ & $x$ & $x$ \\
\hline Coloreda I* & $\mathrm{Cg}$ & $\mathrm{Cg}$ & $\mathrm{Cg}$ & $\mathrm{Cg}, \mathrm{W}$ & $x$ & W & $\mathrm{Cg}, \mathrm{W}$ \\
\hline Corasca & $x$ & $\mathrm{Cg}$ & $x$ & $x$ & $x$ & $x$ & $x$ \\
\hline Coroni & $\mathrm{Cg}$ & $x$ & $x$ & $x$ & $x$ & $x$ & $x$ \\
\hline Cravadora grande I & $\mathrm{Cg}$ & $\mathrm{Cg}$ & $\mathrm{Cg}$ & $\mathrm{Cg}, \mathrm{W}$ & $\mathrm{Cg}$ & W & W \\
\hline Cravadora grande 2 & $\mathrm{Cg}$ & $\mathrm{Og}$ & $x$ & $\mathrm{Cg}, \mathrm{W}$ & $\mathrm{Cg}, \mathrm{W}$ & $\mathrm{Cg}, \mathrm{W}$ & W \\
\hline Cravadora minore I & $\mathrm{Cg}$ & $\mathrm{Cg}$ & $\mathrm{Cg}$ & $x$ & $\mathrm{Cg}$ & $\mathrm{Cg}, \mathrm{W}$ & W \\
\hline Cusanasco & $x$ & $\mathrm{Og}$ & $x$ & $\mathrm{Al}, \mathrm{Vy}, \mathrm{Ar}$ & $x$ & $x$ & $x$ \\
\hline Favà I & $\mathrm{Cg}$ & $\mathrm{Cg}$ & $\mathrm{Cg}$ & $\mathrm{Cg}$ & $\mathrm{Cg}$ & $\mathrm{Cg}$ & $\mathrm{Cg}, \mathrm{W}$ \\
\hline Fosicchia I* & $\mathrm{Cg}, \mathrm{F}$ & $x$ & $x$ & $\mathrm{Cg}, \mathrm{W}$ & $\mathrm{Cg}$ & $x$ & $x$ \\
\hline II Castellaro I & $M$ & Og & Og & Og & $\mathrm{Og}, \mathrm{Cg}$ & $x$ & W \\
\hline Il Castellaro di Bado & $x$ & $x$ & $x$ & $\mathrm{Og}, \mathrm{Cg}$ & $x$ & $x$ & w \\
\hline II Piaggio I & $x$ & Og & $\mathrm{M}, \mathrm{Ar}$ & $\mathrm{Cg}, \mathrm{Al}, \mathrm{W}$ & $M$ & $M$ & W \\
\hline II Piaggio 2 & $x$ & $\mathrm{Og}$ & $\mathrm{Og}, \mathrm{Cg}$ & $\mathrm{Og}, \mathrm{Cg}$ & $\mathrm{Og}, \mathrm{Al}$ & $\mathrm{Al}$ & W \\
\hline II Piaggio 3 & $x$ & $x$ & $x$ & $\mathrm{Og}, \mathrm{Cg}, \mathrm{W}$ & $\mathrm{Cg}$ & $\mathrm{Al}, \mathrm{Vy}$ & W \\
\hline Illegible toponym & $M$ & $x$ & $x$ & $x$ & $x$ & $x$ & $x$ \\
\hline Illegible toponym & $x$ & $\mathrm{Cg}$ & $x$ & $x$ & $x$ & $x$ & $x$ \\
\hline Illegible toponym & $x$ & $x$ & $\mathrm{Al}, \mathrm{Ar}$ & $x$ & $x$ & $x$ & $x$ \\
\hline Illegible toponym & $x$ & $x$ & $\mathrm{Cg}$ & $x$ & $x$ & $x$ & $x$ \\
\hline La Chiappara & $\mathrm{Cg}$ & $x$ & $\mathrm{Cg}$ & x & $\mathrm{Cg}$ & $x$ & $x$ \\
\hline La Chiosa I & $\mathrm{M}, \mathrm{Vy}, \mathrm{Ar}$ & Vy & $\mathrm{Cg}, \mathrm{Ar}, \mathrm{Vy}, \mathrm{Al}$ & $\mathrm{Cg}, \mathrm{Ar}, \mathrm{Vy}, \mathrm{Al}$ & $x$ & $x$ & $\mathrm{~B}, \mathrm{Al}$ \\
\hline La Chiosa 2 & $x$ & $x$ & $x$ & $\mathrm{Cg}$ & $x$ & $x$ & W \\
\hline La dal Canale & $x$ & $x$ & $x$ & $\mathrm{Cg}, \mathrm{W}$ & $\mathrm{Cg}$ & $\mathrm{Al}, \mathrm{Vy}$ & $x$ \\
\hline La Fornace & $x$ & $x$ & $x$ & $\mathrm{Al}, \mathrm{Vy}, \mathrm{Ar}$ & $x$ & $x$ & $x$ \\
\hline La Giara & $H f^{*}$ & $V_{y *}$ & $x$ & $\mathrm{Al}, \mathrm{Vy}, \mathrm{Ar}$ & $x$ & $\mathrm{Al}$ & $x$ \\
\hline La Ligia & $x$ & $\mathrm{x}$ & $x$ & $\mathrm{Cg}, \mathrm{W}$ & $x$ & $\mathrm{~W}, \mathrm{Cg}$ & W \\
\hline La Prata & $x$ & $x$ & $x$ & $\mathrm{Al}, \mathrm{Vy}, \mathrm{Ar}$ & $\mathrm{Al}$ & $x$ & $x$ \\
\hline La Ripalta & $\mathrm{Cg}$ & $x$ & $x$ & $\mathrm{Cg}, \mathrm{W}$ & $x$ & $x$ & $x$ \\
\hline La Riva & $x$ & $x$ & $x$ & $\mathrm{Al}, \mathrm{Vy}, \mathrm{Ar}$ & $\mathrm{Al}, \mathrm{Vy}$ & $x$ & $x$ \\
\hline Linà & $x$ & $\mathrm{Hf}$ & $\mathrm{Al}, \mathrm{Ar}$ & $\mathrm{Cg}$ & $x$ & W & $x$ \\
\hline Linà & $x$ & Og & $x$ & $x$ & $x$ & $x$ & $x$ \\
\hline Madonna del Ponte I* & $x$ & $\mathrm{Cg}$ & $\mathrm{Cg}$ & $\mathrm{Cg}, \mathrm{W}$ & $x$ & w & w \\
\hline Maggioli & $x$ & $x$ & $x$ & $\mathrm{Al}, \mathrm{Vy}, \mathrm{Ar}$ & $x$ & $x$ & $x$ \\
\hline Nel Piano & $M$ & $x$ & $x$ & $\mathrm{x}$ & $\mathrm{Cg}, \mathrm{M}$ & $x$ & $x$ \\
\hline Nel Trezzo & $M$ & $x$ & $x$ & $x$ & $x$ & $x$ & $x$ \\
\hline Patina & $x$ & $x$ & $x$ & $\mathrm{Cg}$ & $x$ & $x$ & $x$ \\
\hline Pezza del Brazo & $x$ & $x$ & $x$ & $\mathrm{Al}, \mathrm{Vy}, \mathrm{Ar}$ & $x$ & $x$ & $x$ \\
\hline Pezza del Molino & x & $x$ & $x$ & $\mathrm{Al}, \mathrm{Vy}, \mathrm{Ar}$ & $x$ & $\mathrm{Al}$ & W \\
\hline Pezzo Grande I* & $\mathrm{M}, \mathrm{Vy}, \mathrm{Ar}$ & $x$ & $x$ & $\mathrm{Al}, \mathrm{Vy}, \mathrm{Ar}$ & $\mathrm{Vy}, \mathrm{M}, \mathrm{Ar}, \mathrm{Al}$ & $x$ & $x$ \\
\hline Pian de Fossa I & $\mathrm{Cg}$ & $\mathrm{Cg}$ & $\mathrm{Cg}$ & $\mathrm{Cg}, \mathrm{W}$ & $\mathrm{Cg}$ & $\mathrm{Cg}$ & W \\
\hline Piè di Favà & $M$ & $x$ & $\mathrm{M}, \mathrm{Ar}$ & $x$ & $x$ & $x$ & W \\
\hline Piè di Favà & $M^{*}$ & $x$ & $\mathrm{M}, \mathrm{Ar}$ & $\mathrm{x}$ & $x$ & x & W \\
\hline Pignon soprano I & $M, V_{y}, A r$ & $\mathrm{Hf}$ & $\mathrm{Cg}, \mathrm{Ar}, \mathrm{Vy}, \mathrm{Al}$ & $\mathrm{Cg}, \mathrm{Ar}, \mathrm{Vy}, \mathrm{Al}$ & $x$ & $\mathrm{Al}, \mathrm{Vy}, \mathrm{Cg}$ & $x$ \\
\hline Pignon soprano 2 & $\mathrm{x}$ & $x$ & $\mathrm{Cg}, \mathrm{Ar}, \mathrm{Vy}, \mathrm{Al}$ & $x$ & $x$ & $\mathrm{Al}, \mathrm{Vy}, \mathrm{Cg}$ & $x$ \\
\hline Pignon soprano 3 & $x$ & $x$ & $\mathrm{Cg}, \mathrm{Ar}, \mathrm{Vy}, \mathrm{Al}$ & $x$ & $x$ & $x$ & $x$ \\
\hline Pignon soprano 4 & $x$ & $x$ & $\mathrm{Cg}, \mathrm{Ar}, \mathrm{Vy}, \mathrm{Al}$ & $x$ & $x$ & $x$ & $x$ \\
\hline Pignon soprano 5 & $x$ & $x$ & $\mathrm{Cg}, \mathrm{Ar}, \mathrm{Vy}, \mathrm{Al}$ & $x$ & $\mathrm{Al}$ & $x$ & $x$ \\
\hline
\end{tabular}


Table I: Inventories (part A) (Continued)

\begin{tabular}{|c|c|c|c|c|c|c|c|}
\hline Pignon soprano 6 & $x$ & $x$ & $M$ & $x$ & $M$ & $x$ & $x$ \\
\hline Pignon soprano 7 & $x$ & $x$ & $\mathrm{Og}, \mathrm{Cg}$ & $\mathrm{x}$ & $x$ & $x$ & $x$ \\
\hline Pilora I & $x$ & $x$ & $x$ & $\mathrm{Al}, \mathrm{Vy}, \mathrm{Ar}$ & $x$ & $\mathrm{Og}$ & Og \\
\hline Postemi & $x$ & $\mathrm{Cg}$ & $x$ & $\mathrm{x}$ & $x$ & $x$ & $x$ \\
\hline Rezzo I & $\mathrm{Cg}$ & $x$ & $\mathrm{Ar}$ & $x$ & $x$ & $x$ & $x$ \\
\hline Rezzo 2 & $\mathrm{G}$ & $x$ & $x$ & $x$ & $x$ & $x$ & $x$ \\
\hline Rezzo 3 & $\mathrm{G}^{*}$ & $V_{y *}$ & $x$ & $x$ & $x$ & $x$ & $x$ \\
\hline Rezzo 4 & $M$ & $x$ & $\mathrm{Ar}$ & $x$ & $x$ & $x$ & $x$ \\
\hline Rezzo 5 & $M$ & $x$ & $\mathrm{Ar}$ & $x$ & $x$ & $x$ & $x$ \\
\hline S. Antonio I & $x$ & $x$ & $x$ & Al, $\mathrm{Vy}, \mathrm{Ar}$ & $x$ & Og & $\mathrm{W}, \mathrm{Og}$ \\
\hline S. Michele & $x$ & $x$ & $\mathrm{M}, \mathrm{Ar}$ & $\mathrm{Al}, \mathrm{Vy}, \mathrm{Ar}$ & $x$ & $x$ & $x$ \\
\hline Scandolara I* & $\mathrm{Cg}$ & $\mathrm{Cg}$ & $\mathrm{Cg}$ & x & $x$ & $\mathrm{Cg}$ & w \\
\hline Sotto la Lama & $x$ & $x$ & $x$ & $\mathrm{Al}, \mathrm{Vy}, \mathrm{Ar}$ & $x$ & $x$ & $x$ \\
\hline Spinzo I & $x$ & $\mathrm{Og}$ & $x$ & x & $x$ & $x$ & $x$ \\
\hline Spinzo 2 & $x$ & $\mathrm{Og}$ & $x$ & $x$ & $x$ & $x$ & $x$ \\
\hline Spinzo 3 & $x$ & $\mathrm{Og}$ & $x$ & $x$ & $x$ & $x$ & $x$ \\
\hline Sprugola A & $\mathrm{Cg}$ & $x$ & $\mathrm{Cg}, \mathrm{M}$ & $x$ & $x$ & $x$ & $x$ \\
\hline Sprugola B & $\mathrm{Cg}$ & $x$ & $\mathrm{Cg}$ & $x$ & $x$ & $x$ & $x$ \\
\hline Su dal Canale & $x$ & $x$ & $x$ & $\mathrm{Cg}, \mathrm{W}$ & $x$ & $x$ & $x$ \\
\hline Tra la Chiesa & $M$ & $x$ & $\mathrm{Og}, \mathrm{Cg}$ & $x$ & $x$ & $x$ & $\mathrm{~W}, \mathrm{Vy}$ \\
\hline Tra le case & $M$ & $\mathrm{Hf}$ & $M$ & $x$ & $x$ & $x$ & $\mathrm{x}$ \\
\hline$V a^{\prime} I$ & $x$ & $x$ & $x$ & $\mathrm{Cg}$ & $\mathrm{Cg}$ & $\mathrm{Cg}$ & $x$ \\
\hline Vaccheredo & $\mathrm{Cg}$ & $x$ & $x$ & $x$ & $x$ & $x$ & $x$ \\
\hline
\end{tabular}

Inventories, ordered for every toponym, of the Parish of St. Mary and of the Municipality of Pignone, for the years 1588, 1663, 1763, 1828, 1878, 1933 and recent times. Part A comprises toponyms listed in the ecclesiastic inventories (years 1588, 1663, 1763, 1828). Legend: Ar= arboretum; B = buildings; $\mathrm{Cg}=$ chestnut grove; $\mathrm{Hf}=$ hempfield; $\mathrm{G}=$ grassland; $\mathrm{M}=$ meadow; $\mathrm{Og}=$ olive grove; $\mathrm{Al}=$ arable land; $\mathrm{W}=$ woodland; $\mathrm{Vy}=$ vineyard; $*$ = include a mill; $\mathbf{x}$ : no data.

Pignone. The former are stored in the "Niccolo V" Lunense Episcopal Archive of Sarzana (SP): series "Filze Parrocchiali" [12-15]. The latter are stored in the State Archive of La Spezia $[16,17]$.

Analysis of aerial photographs and field surveys allowed the investigation of current land use (crops, natural vegetation, buildings). Ancient toponyms listed in the inventories were compared with current ones that can be found in the cadastral map of the Municipality of Pignone. A complete correlation between ancient and current toponyms does not exist: the former, in part, have been lost; in some cases, more than one type of crop exists for a single toponym.

The antique inventories were written in ancient Italian. Hence the following words, which were found singly or together and linked to toponyms or individually, were annotated (Table 1, Table 2, Figure 2):

- Arboretum (Ar): field planted, probably with fruit trees, such as walnut (Juglans regia L.), fig (Ficus carica L.), apple (Malus domestica (Borkh.) Borkh.), etc. [18,19];

- Buildings (B): area occupied by recent buildings (houses, parking, ways, etc);

- Chestnut grove (Cg): anthropogenic woods of chestnut (Castanea sativa), frequently in terrace cultivation.
- Meadow (M): land, probably held for forage production;

- Grassland (G);

- Hempfield (Hf): farmland for hemp (Cannabis sativa L.);

- Olive grove $(\mathrm{Og})$ : land planted with olives (Olea europaea L.), often in terrace cultivation;

- Woodland (W): vegetation to forest (Quercus spp., Pinus pinaster, Ostrya carpinifolia, Fraxinus ornus, Salix spp., etc.);

- Arable land (Al): very probably crops with wheat (Triticum spp.), barley (Hordeum vulgare L.) and rye (Secale cereale L.); in more recent times, maize (Zea mays L.) or vegetables, such as broad bean (Vicia faba L.), pea (Pisum sativum L.) and chick pea (Cirer arietinum L.) [18,19].

- Vineyard (Vy): land planted with vines (Vitis vinifera L.).

The percent frequency of all crop portions (type) were computed for each inventory (Figure 2). The relationship between the total number of fields (referred to as toponyms) and the total number of crop portions (for all fields) have been calculated for each inventory as an indicator of crop uniformity (CU).

The botanical nomenclature used in this paper follows [20]. 
Table 2: Inventories (part B)

\begin{tabular}{|c|c|c|c|c|c|c|c|}
\hline \multicolumn{8}{|c|}{ Type of crop } \\
\hline Toponym & 1588 & 1663 & 1763 & 1828 & 1878 & 1933 & 2008 \\
\hline Ai Laghi I & $x$ & $x$ & $x$ & $x$ & $W$ & $\mathrm{Al}$ & $\mathrm{Al}$ \\
\hline Ai Laghi 2 & $x$ & $x$ & $x$ & $x$ & $x$ & $\mathrm{Al}, \mathrm{Vy}$ & $\mathrm{Al}, \mathrm{Vy}$ \\
\hline Alle Noci & $x$ & $x$ & $x$ & $x$ & $x$ & W & W \\
\hline Bandita & $x$ & $x$ & $x$ & $x$ & $x$ & $w$ & $w$ \\
\hline Banzola I & $x$ & $x$ & $x$ & $x$ & $\mathrm{Cg}$ & $\mathrm{W}, \mathrm{Cg}$ & W \\
\hline Banzola 2 & $x$ & $x$ & $x$ & $x$ & W & $w^{\circ}$ & W \\
\hline Banzola 3 & $x$ & $x$ & $x$ & $x$ & W & w & W \\
\hline Banzola 4 & $x$ & $x$ & $x$ & $x$ & $x$ & W & W \\
\hline Battipagliano I & $x$ & $x$ & $x$ & $x$ & $\mathrm{Cg}$ & $\mathrm{Cg}$ & $\mathrm{Cg}$ \\
\hline Battipagliano 2 & $x$ & $x$ & $x$ & $x$ & $\mathrm{Al}$ & Al & $x$ \\
\hline Battipagliano 3 & $x$ & $x$ & $x$ & $x$ & $\mathrm{Al}, \mathrm{Cg}$ & $\mathrm{Cg}, \mathrm{M}$ & $\mathrm{Cg}, \mathrm{W}$ \\
\hline Battipagliano 4 & $x$ & $x$ & $x$ & $x$ & $\mathrm{Vy}, \mathrm{Al}$ & $\mathrm{Al}$ & W \\
\hline Battipagliano 5 & $x$ & $x$ & $x$ & $x$ & $\mathrm{Vy}, \mathrm{Al}$ & $\mathrm{Al}$ & W \\
\hline Battipagliano 6 & $x$ & $x$ & $x$ & $x$ & $x$ & $\mathrm{Al}$ & $\mathrm{Al}$ \\
\hline Battipagliano 7 & $x$ & $x$ & $x$ & $x$ & $x$ & $\mathrm{Al}, \mathrm{M}$ & $\mathrm{Al}, \mathrm{M}$ \\
\hline Bogiolo & $x$ & $\mathrm{x}$ & $x$ & $x$ & $x$ & $\mathrm{Al}$ & $\mathrm{Al}$ \\
\hline Borale I & $x$ & $x$ & $x$ & $x$ & Vy, Al & $\mathrm{Al}$ & $\mathrm{Al}$ \\
\hline Borale 2 & $x$ & $x$ & $x$ & $x$ & Vy, Al, Og & $x$ & w \\
\hline Borale 3 & $x$ & $x$ & $x$ & $x$ & $\mathrm{Vy}, \mathrm{Al}$ & $x$ & W \\
\hline Borale 4 & $x$ & $x$ & $x$ & $x$ & $\mathrm{Al}, \mathrm{Vy}$ & $x$ & $M$ \\
\hline Borale 5 & $x$ & $x$ & $x$ & $x$ & $\mathrm{Cg}$ & $x$ & $B, W$ \\
\hline Bosco del Gallino & $x$ & $x$ & $x$ & $x$ & W & $x$ & W \\
\hline Calcinarla 3 & $x$ & $x$ & $x$ & $x$ & w & $x$ & $\mathrm{~B}, \mathrm{Al}$ \\
\hline Calcinarla 4 & $x$ & $x$ & $x$ & $x$ & $A l, V y$, irrig & $\mathrm{Al}$ & $\mathrm{B}, \mathrm{Al}$ \\
\hline Calcinarla 5 & $x$ & $x$ & $x$ & $x$ & $\mathrm{Cg}$ & $x$ & $\mathrm{~B}, \mathrm{Al}$ \\
\hline Calcinarla 6 & $x$ & $x$ & $x$ & $x$ & $\mathrm{Al}, \mathrm{Vy}$ & $\mathrm{Al}, \mathrm{Vy}, \mathrm{Cg}$ & $\mathrm{B}, \mathrm{Al}$ \\
\hline Calcinarla 7 & $x$ & $x$ & $x$ & $x$ & $\mathrm{Al}, \mathrm{Vy}$ & $\mathrm{Al}$ & $\mathrm{B}, \mathrm{Al}$ \\
\hline Calcinarla 8 & $x$ & $x$ & $x$ & $x$ & $\mathrm{Al}, \mathrm{Vy}_{\mathrm{y}}$ & $x$ & $\mathrm{~B}, \mathrm{Al}$ \\
\hline Calcinarla 9 & $x$ & $x$ & $x$ & $x$ & $\mathrm{Al}, \mathrm{Vy}$ & $x$ & $\mathrm{~B}, \mathrm{Al}$ \\
\hline Campuscasco 2 & $x$ & $x$ & $x$ & $x$ & $\mathrm{Cg}$ & $x$ & $w$ \\
\hline Cantarana & $x$ & $x$ & $x$ & $x$ & $\mathrm{Al}, \mathrm{Vy}$ & $x$ & $x$ \\
\hline Castellino & $x$ & $x$ & $x$ & $x$ & $x$ & w & $x$ \\
\hline Cerreta & $x$ & $x$ & $x$ & $x$ & $x$ & $\mathrm{Cg}, \mathrm{W}$ & $x$ \\
\hline Cerreta & $x$ & $x$ & $x$ & $x$ & Og & W & $x$ \\
\hline Ciazzo & $x$ & $x$ & $x$ & $x$ & $\mathrm{Vy}, \mathrm{Al}$ & $x$ & $x$ \\
\hline Colorede 2 & $x$ & $x$ & $x$ & $x$ & W & w & $\mathrm{Cg}, \mathrm{W}$ \\
\hline Costa dell'Olivella & $x$ & $x$ & $x$ & $x$ & $\mathrm{Cg}$ & $x$ & W \\
\hline Cravadora I & $x$ & $x$ & $x$ & $x$ & $\mathrm{Cg}$ & $\mathrm{Cg}, \mathrm{W}$ & $w$ \\
\hline Cravadora 2 & $x$ & $x$ & $x$ & $x$ & W & W & W \\
\hline Cravadora 3 & $x$ & $x$ & $x$ & $x$ & $\mathrm{Cg}$ & $\mathrm{Cg}$ & w \\
\hline Cravadora 4 & $x$ & $x$ & $x$ & $x$ & $\mathrm{Cg}$ & $W$ & W \\
\hline Cravadora 5 & $x$ & $x$ & $x$ & $x$ & $\mathrm{Cg}$ & w & W \\
\hline Cravadora 6 & $x$ & $x$ & $x$ & $x$ & $\mathrm{Cg}$ & w & w \\
\hline Cravadora 7 & $x$ & $x$ & $x$ & $x$ & $\mathrm{Cg}$ & $\mathrm{Cg}, \mathrm{W}$ & W \\
\hline Cravadora di Sotto & $x$ & $x$ & $x$ & $x$ & $\mathrm{~W}, \mathrm{Cg}$ & W & $\mathrm{W}, \mathrm{Cg}$ \\
\hline Cravadora minore 2 & $x$ & $x$ & $x$ & $x$ & $\mathrm{Cg}$ & w & $W^{0}$ \\
\hline Cuccaro I & $x$ & $x$ & $x$ & $x$ & $\mathrm{Al}, \mathrm{Vy}, \mathrm{Ar}$ & $W$ & W \\
\hline Cuccaro 10 & $x$ & $x$ & $x$ & $x$ & $\mathrm{Cg}$ & $\mathrm{Cg}$ & $\mathrm{Cg}$ \\
\hline Cuccaro 2 & $x$ & $x$ & $x$ & $x$ & $\mathrm{Al}$ & W & W \\
\hline Cuccaro 3 & $x$ & $x$ & $x$ & $x$ & $\mathrm{Cg}$ & W & $\mathrm{W}, \mathrm{Cg}$ \\
\hline Cuccaro 4 & $x$ & $x$ & $x$ & $x$ & $\mathrm{Al}, \mathrm{Vy}, \mathrm{Ar}$ & $\mathrm{Al}, \mathrm{Vy}, \mathrm{Cg}$ & $\mathrm{Al}$ \\
\hline Cuccaro 5 & $x$ & $x$ & $x$ & $x$ & Al & $\mathrm{Al}, \mathrm{Vy}$ & Vy \\
\hline Cuccaro 6 & $x$ & $x$ & $x$ & $x$ & $\mathrm{Al}$ & W & W \\
\hline Cuccaro 7 & $x$ & $x$ & $x$ & $x$ & $\mathrm{Cg}$ & $\mathrm{Cg}, \mathrm{W}$ & W \\
\hline Cuccaro 8 & $x$ & $x$ & $x$ & $x$ & $\mathrm{Al}, \mathrm{Vy}$ & $\mathrm{Al}, \mathrm{Vy}$ & $\mathrm{Al}, \mathrm{Vy}$ \\
\hline Cuccaro 9 & $x$ & $x$ & $x$ & $x$ & $\mathrm{Al}$ & W & W \\
\hline Cuneo I & $x$ & $x$ & $x$ & $x$ & $x$ & $\mathrm{Al}, \mathrm{W}$ & $\mathrm{Al}, \mathrm{W}$ \\
\hline
\end{tabular}


Table 2: Inventories (part B) (Continued)

\begin{tabular}{|c|c|c|c|c|c|c|c|}
\hline Cuneo 2 & $x$ & $x$ & $x$ & $x$ & $x$ & W & $x$ \\
\hline Cuneo sottano I & $x$ & $x$ & $x$ & $x$ & $x$ & $\mathrm{Al}$ & $x$ \\
\hline Cuneo sottano 2 & $x$ & $x$ & $x$ & $x$ & $x$ & $\mathrm{~W}, \mathrm{Al}$ & $x$ \\
\hline Cusidore & $x$ & $x$ & $x$ & $x$ & W & $x$ & W \\
\hline Due Canali I & $x$ & $x$ & $x$ & $x$ & $x$ & $\mathrm{Cg}$ & $x$ \\
\hline Due Canali 2 & $x$ & $x$ & $x$ & $x$ & $x$ & $\mathrm{Cg}$ & $x$ \\
\hline Due Canali 3 & $x$ & $x$ & $x$ & $x$ & $x$ & $\mathrm{Cg}$ & $x$ \\
\hline Favà 2 & $x$ & $x$ & $x$ & $x$ & $\mathrm{Cg}, \mathrm{Og}$ & $\mathrm{Cg}$ & $\mathrm{Cg}, \mathrm{W}$ \\
\hline Favà 3 & $x$ & $x$ & $x$ & $x$ & $\mathrm{Cg}, \mathrm{Og}$ & W & W \\
\hline Filagne & $x$ & $x$ & $x$ & $x$ & $\mathrm{Al}$ & $x$ & $x$ \\
\hline Fontanella I & $x$ & $x$ & $x$ & $x$ & $\mathrm{Cg}, \mathrm{W}$ & $x$ & $\mathrm{Cg}, \mathrm{W}$ \\
\hline Fontanella 2 & $x$ & $x$ & $x$ & $x$ & $\mathrm{Cg}, \mathrm{W}$ & $x$ & $\mathrm{Cg}, \mathrm{W}$ \\
\hline Fontanella 3 & $x$ & $x$ & $x$ & $x$ & W & $x$ & W \\
\hline Fosicchia $2^{*}$ & $x$ & $x$ & $x$ & $x$ & $\mathrm{Cg}$ & $\mathrm{Cg}$ & $x$ \\
\hline Fosicchia $3 *$ & $x$ & $x$ & $x$ & $x$ & $\mathrm{Cg}$ & $\mathrm{Cg}$ & $x$ \\
\hline Fossalonga & $x$ & $x$ & $x$ & $x$ & $\mathrm{Cg}$ & $x$ & $x$ \\
\hline Foxina I & $x$ & $x$ & $x$ & $x$ & M & $\mathrm{Al}$ & $x$ \\
\hline Foxina 2 & $x$ & $x$ & $x$ & $x$ & $\mathrm{Al}, \mathrm{Vy}$ & $\mathrm{Al}$ & $x$ \\
\hline Foxina 3 & $x$ & $x$ & $x$ & $x$ & $\mathrm{x}$ & $\mathrm{Al}$ & $x$ \\
\hline Foxina 4 & $x$ & $x$ & $x$ & $x$ & $x$ & $\mathrm{Al}$ & $x$ \\
\hline Foxina Grande & $x$ & $x$ & $x$ & $x$ & $x$ & $\mathrm{Al}, \mathrm{Vy}$ & $x$ \\
\hline Frantoio I & $x$ & $x$ & $x$ & $x$ & $x$ & $\mathrm{Al}, \mathrm{Vy}$ & $x$ \\
\hline Frantoio 2 & $\hat{x}$ & $\hat{x}$ & $\hat{x}$ & $x$ & $\hat{x}$ & $\mathrm{Al}, \mathrm{Vy}$ & $\hat{x}$ \\
\hline Frantoio 3 & $x$ & $x$ & $x$ & $x$ & $x$ & $\mathrm{Al}$ & $x$ \\
\hline Frantoio 4 & $x$ & $x$ & $x$ & $x$ & $x$ & $\mathrm{Al}$ & $x$ \\
\hline Frassaneda & $x$ & $x$ & $x$ & $x$ & $x$ & W & $x$ \\
\hline Gaggiolo & $x$ & $x$ & $x$ & $x$ & $\mathrm{Cg}, \mathrm{Al}$ & w & $x$ \\
\hline Garzarelli & $x$ & $x$ & $x$ & $x$ & $x$ & W & $x$ \\
\hline Grizzola I & $x$ & $x$ & $x$ & $x$ & $\mathrm{Vy}, \mathrm{Ar}, \mathrm{Al}$ & W, Cg & $\mathrm{W}, \mathrm{Cg}$ \\
\hline Grizzola 2 & $x$ & $x$ & $x$ & $x$ & $\mathrm{Cg}, \mathrm{Og}$ & $\mathrm{Al}, \mathrm{Vy}, \mathrm{Og}, \mathrm{Cg}$ & $\mathrm{W}, \mathrm{Cg}$ \\
\hline Grizzola 3 & $x$ & $x$ & $x$ & $x$ & $\mathrm{Al}$ & $\mathrm{Cg}$ & $\mathrm{W}, \mathrm{Cg}$ \\
\hline Grizzola 4 & $x$ & $x$ & $x$ & $x$ & $\mathrm{Cg}$ & $\mathrm{Cg}$ & $\mathrm{W}, \mathrm{Cg}$ \\
\hline Grizzola 5 & $x$ & $x$ & $x$ & $x$ & $\mathrm{Cg}$ & $\mathrm{Cg}$ & $\mathrm{W}, \mathrm{Cg}$ \\
\hline Grizzola 6 & $\hat{x}$ & $\hat{x}$ & $\hat{x}$ & $x$ & $\mathrm{Cg}, \mathrm{Og}, \mathrm{Al}$ & $\mathrm{Cg}$ & W \\
\hline Grizzola 7 & $x$ & $x$ & $x$ & $x$ & $x$ & $\mathrm{Al}, \mathrm{Vy}, \mathrm{Og}$ & $\mathrm{Al}, \mathrm{Vy}, \mathrm{Og}$ \\
\hline II Campo I & $x$ & $x$ & $x$ & $x$ & $\mathrm{Vy}, \mathrm{Ar}, \mathrm{Al}$ & $\mathrm{Al}$ & $\mathrm{x}$ \\
\hline II Campo 2 & $x$ & $x$ & $x$ & $x$ & $\mathrm{Vy}, \mathrm{Ar}, \mathrm{Al}$ & $\mathrm{Al}, \mathrm{Vy}$ & $x$ \\
\hline II Campo 3 & $x$ & $x$ & $x$ & $x$ & $\mathrm{Al}$ & $\mathrm{Al}$ & $x$ \\
\hline II Campo 4 & $x$ & $x$ & $x$ & $x$ & $\mathrm{Vy}, \mathrm{Al}, \mathrm{Cg}$ & $\mathrm{Cg}$ & $x$ \\
\hline II Castellaro 2 & $x$ & $x$ & $x$ & $x$ & $\mathrm{Og}$ & $x$ & W \\
\hline II Giardino & $x$ & $x$ & $x$ & $x$ & $\mathrm{Al}, \mathrm{Vy}$ & $x$ & $x$ \\
\hline II Piaggio 4 & $\hat{x}$ & $\hat{x}$ & $\hat{x}$ & $x$ & $\mathrm{Al}$ & $\hat{\mathrm{Al}}$ & $\hat{x}$ \\
\hline II Piaggio 5 & $x$ & $x$ & $x$ & $x$ & $x$ & $\mathrm{Al}$ & $x$ \\
\hline II Piaggio 6 & $x$ & $x$ & $x$ & $x$ & $x$ & $\mathrm{Og}, \mathrm{Al}$ & $x$ \\
\hline Lama di Pianello & $\hat{x}$ & $\hat{x}$ & $\hat{x}$ & $\hat{x}$ & $\mathrm{Cg}, \mathrm{W}$ & $\mathrm{x}$ & $\hat{x}$ \\
\hline Madonna del Ponte $2 *$ & $x$ & $x$ & $x$ & $x$ & $\mathrm{Cg}$ & $\mathrm{Cg}$ & W \\
\hline Madonna del Ponte 3* & $x$ & $x$ & $x$ & $x$ & $\mathrm{Cg}$ & $x$ & $\mathrm{~W}, \mathrm{Cg}$ \\
\hline Migiaro & $x$ & $x$ & $x$ & $x$ & $\mathrm{Cg}$ & $x$ & $\mathrm{~W}, \mathrm{Cg}$ \\
\hline Migliarese I & $x$ & $x$ & $x$ & $x$ & $\mathrm{Cg}$ & $\mathrm{Cg}$ & $\mathrm{W}, \mathrm{Cg}$ \\
\hline Migliarese 2 & $x$ & $x$ & $x$ & $x$ & $\mathrm{Cg}$ & $\mathrm{Cg}$ & $\mathrm{W}, \mathrm{Cg}$ \\
\hline Migliarese 3 & $x$ & $x$ & $x$ & $x$ & $x$ & W & W \\
\hline Migliarese 4 & $x$ & $x$ & $x$ & $x$ & $x$ & $\mathrm{Cg}$ & W \\
\hline Migliarese 5 & $x$ & $x$ & $x$ & $x$ & $x$ & $\mathrm{Cg}$ & w \\
\hline Migliarese 6 & $x$ & $x$ & $x$ & $x$ & $x$ & W & w \\
\hline Migliarese 7 & $x$ & $x$ & $x$ & $x$ & $x$ & w & W \\
\hline Migliarese 8 & $\hat{x}$ & $\hat{x}$ & $\hat{x}$ & $\hat{x}$ & $x$ & W & w \\
\hline Migliarese di sopra & $\hat{x}$ & $\hat{x}$ & $\hat{x}$ & $x$ & $x$ & W & w \\
\hline Migliarese di sotto & $x$ & $x$ & $x$ & $x$ & x & $\mathrm{Cg}, \mathrm{W}$ & w \\
\hline Monteletto I & $x$ & $x$ & $x$ & $x$ & Og, $\mathrm{Vy}, \mathrm{Al}$ & $M$ & $x$ \\
\hline Monteletto 2 & $x$ & $x$ & $x$ & $x$ & $\mathrm{Og}, \mathrm{Vy}, \mathrm{Al}$ & $x$ & $x$ \\
\hline Monteletto 3 & $x$ & $x$ & $x$ & $x$ & $\mathrm{Vy}, \mathrm{Og}, \mathrm{Cg}$ & $x$ & $x$ \\
\hline Monteletto 4 & $\hat{x}$ & $\hat{x}$ & $\hat{x}$ & $\hat{x}$ & $\mathrm{Cg}$ & $\hat{x}$ & $x$ \\
\hline Mostani & $\hat{x}$ & $\hat{x}$ & $\hat{x}$ & $x$ & $\mathrm{Cg}, \mathrm{W}$ & $\hat{x}$ & $x$ \\
\hline
\end{tabular}


Table 2: Inventories (part B) (Continued)

\begin{tabular}{|c|c|c|c|c|c|c|c|}
\hline Narneia Grande I & $x$ & $x$ & $x$ & $x$ & $x$ & W & $x$ \\
\hline Narneia Grande 2 & $x$ & $x$ & $x$ & $x$ & $x$ & W & $x$ \\
\hline Olivella & $x$ & $x$ & $x$ & $x$ & $\mathrm{Cg}$ & $x$ & W \\
\hline Orto I & $x$ & $x$ & $x$ & $x$ & $x$ & $\mathrm{Al}$ & $x$ \\
\hline Orto 2 & $x$ & $x$ & $x$ & $x$ & $x$ & $\mathrm{Al}, \mathrm{Vy}$ & $x$ \\
\hline Orto 3 & $x$ & $x$ & $x$ & $x$ & $x$ & $\mathrm{Al}, \mathrm{Vy}$ & $x$ \\
\hline Pastine I & $x$ & $x$ & $x$ & $x$ & $\mathrm{Al}, \mathrm{Vy}$ & $\mathrm{Al}, \mathrm{Vy}$ & $\mathrm{Al}, \mathrm{Vy}$ \\
\hline Pastine 2 & $x$ & $x$ & $x$ & $x$ & $\mathrm{Cg}$ & $\mathrm{Vy}, \mathrm{Cg}$ & $\mathrm{Cg}$ \\
\hline Pastine 3 & $x$ & $x$ & $x$ & $x$ & $\mathrm{Cg}$ & $\mathrm{Vy}, \mathrm{Al}$ & $\mathrm{Vy}, \mathrm{Al}$ \\
\hline Pastine 4 & $x$ & $x$ & $x$ & $x$ & $\mathrm{Cg}, \mathrm{W}$ & $\mathrm{x}$ & $\mathrm{Cg}, \mathrm{W}$ \\
\hline Pastine 5 & $x$ & $x$ & $x$ & $x$ & $\mathrm{Vy}, \mathrm{Ar}, \mathrm{Al}$ & $\mathrm{x}$ & $\mathrm{Cg}, \mathrm{W}$ \\
\hline Pastine 6 & $x$ & $x$ & $x$ & $x$ & $x$ & $\mathrm{Al}, \mathrm{Vy}, \mathrm{Cg}$ & $\mathrm{Cg}, \mathrm{W}$ \\
\hline Perlo I & $x$ & $x$ & $x$ & $x$ & $\mathrm{Og}, \mathrm{Cg}$ & $\mathrm{Og}, \mathrm{Cg}$ & W \\
\hline Perlo 2 & $x$ & $x$ & $x$ & $x$ & $x$ & $\mathrm{Al}$ & $\mathrm{W}, \mathrm{Cg}$ \\
\hline Perlo 3 & $x$ & $x$ & $x$ & $x$ & $x$ & $W, C g$ & W, Cg \\
\hline Perlo 4 & $x$ & $x$ & $x$ & $x$ & $x$ & $\mathrm{Al}, \mathrm{Cg}$ & $w^{\circ}$ \\
\hline Perlo 5 & $x$ & $x$ & $x$ & $x$ & $x$ & Og & W \\
\hline Perlo 6 & $x$ & $x$ & $x$ & $x$ & $x$ & $\mathrm{Al}, \mathrm{Og}, \mathrm{Cg}$ & w \\
\hline Pezzo Grande 2* & $x$ & $x$ & $x$ & $x$ & $\mathrm{Vy}, \mathrm{Al}$ & $\mathrm{x}$ & $x$ \\
\hline Pian de Fossa 10 & $x$ & $x$ & $x$ & $x$ & $x$ & $\mathrm{Cg}$ & W \\
\hline Pian de Fossa 2 & $x$ & $x$ & $x$ & $x$ & $x$ & $\mathrm{Cg}$ & $\mathrm{W}, \mathrm{Cg}$ \\
\hline Pian de Fossa 3 & $x$ & $x$ & $x$ & $x$ & $\mathrm{Al}$ & $\mathrm{Cg}$ & $\mathrm{W}, \mathrm{Cg}$ \\
\hline Pian de Fossa 4 & $x$ & $x$ & $x$ & $x$ & $\mathrm{Al}, \mathrm{Cg}$ & $\mathrm{Al}, \mathrm{Vy}, \mathrm{Cg}$ & $w^{\circ}$ \\
\hline Pian de Fossa 5 & $x$ & $x$ & $x$ & $x$ & $\mathrm{Cg}$ & $\mathrm{Cg}$ & W \\
\hline Pian de Fossa 6 & $x$ & $x$ & $x$ & $x$ & $\mathrm{Cg}$ & $\mathrm{Cg}$ & W \\
\hline Pian de Fossa 7 & $x$ & $x$ & $x$ & $x$ & $\mathrm{Cg}$ & $\mathrm{Cg}$ & W \\
\hline Pian de Fossa 8 & $x$ & $x$ & $x$ & $x$ & $\mathrm{Cg}$ & $\mathrm{Cg}$ & w \\
\hline Pian de Fossa 9 & $x$ & $x$ & $x$ & $x$ & $x$ & $\mathrm{Og}$ & W \\
\hline Pian di Casa & $x$ & $x$ & $x$ & $x$ & $x$ & $\mathrm{Al}$ & $x$ \\
\hline Pian di Manin & $x$ & $x$ & $x$ & $x$ & $x$ & $\mathrm{Al}, \mathrm{M}$ & $x$ \\
\hline Piana di Gatella & $x$ & $x$ & $x$ & $x$ & $\mathrm{Cg}$ & $x$ & $x$ \\
\hline Piano del Ponte rotto & $x$ & $x$ & $x$ & $x$ & $x$ & $\mathrm{Al}$ & $x$ \\
\hline Piazzale & $x$ & $x$ & $x$ & $x$ & $\mathrm{Vy}, \mathrm{Al}, \mathrm{Cg}$ & $\mathrm{Vy}, \mathrm{Al}$ & $x$ \\
\hline Pignon sottano I & $x$ & $x$ & $x$ & $x$ & $\mathrm{Al}$ & $\mathrm{Al}$ & $x$ \\
\hline Pignon sottano 2 & $x$ & $x$ & $x$ & $x$ & $\mathrm{Vy}, \mathrm{Ar}, \mathrm{Al}$ & $x$ & $x$ \\
\hline Pignon sottano 3 & $x$ & $x$ & $x$ & $x$ & Al, Vy & $\mathrm{Al}, \mathrm{Vy}$ & $x$ \\
\hline Pignon sottano 4 & $x$ & $x$ & $x$ & $x$ & $\mathrm{Vy}, \mathrm{Ar}, \mathrm{Al}$ & $\mathrm{x}$ & $x$ \\
\hline Pignon sottano 5 & $x$ & $x$ & $x$ & $x$ & $\mathrm{Vy}, \mathrm{Al}$ & $\mathrm{Vy}, \mathrm{Al}$ & $x$ \\
\hline Pignon sottano 6 & $x$ & $x$ & $x$ & $x$ & $\mathrm{Vy}, \mathrm{Al}$ & $\mathrm{Vy}, \mathrm{Al}$ & $x$ \\
\hline Pignon sottano 7 & $x$ & $x$ & $x$ & $x$ & $\mathrm{Vy}, \mathrm{Al}$ & $\mathrm{Vy}, \mathrm{Al}$ & $x$ \\
\hline Pignon sottano 8 & $x$ & $x$ & $x$ & $x$ & $\mathrm{Vy}, \mathrm{Al}$ & $x$ & $x$ \\
\hline Pilora 2 & $x$ & $x$ & $x$ & $x$ & $x$ & Og & W \\
\hline Pilora 3 & $x$ & $x$ & $x$ & $x$ & $x$ & $\mathrm{Og}$ & w \\
\hline Pilora 4 & $x$ & $x$ & $x$ & $x$ & $x$ & $\mathrm{Al}$ & W \\
\hline Porcile & $x$ & $x$ & $x$ & $x$ & $\mathrm{Vy}, \mathrm{M}, \mathrm{Ar}, \mathrm{Al}$ & $x$ & $x$ \\
\hline Porcinasco & $x$ & $x$ & $x$ & $x$ & $\mathrm{Al}$ & $x$ & $x$ \\
\hline Possessione I & $x$ & $x$ & $x$ & $x$ & $\mathrm{Al}$ & $\mathrm{Al}, \mathrm{Vy}, \mathrm{Cg}$ & $\mathrm{Al}, \mathrm{Vy}, \mathrm{Cg}$ \\
\hline Possessione 2 & $x$ & $x$ & $x$ & $x$ & $x$ & $\mathrm{Al}, \mathrm{Vy}$ & $\mathrm{Al}, \mathrm{Vy}$ \\
\hline Possessione 3 & $x$ & $x$ & $x$ & $x$ & x & $\mathrm{Al}, \mathrm{Vy}$ & $\mathrm{Al}, \mathrm{Vy}$ \\
\hline Pradiera I & $x$ & $x$ & $x$ & $x$ & $\mathrm{Ar}, \mathrm{Vy}, \mathrm{Al}$ & $x$ & $x$ \\
\hline Pradiera 2 & $x$ & $x$ & $x$ & $x$ & $\mathrm{Vy}, \mathrm{Al}$ & $x$ & $x$ \\
\hline Pradiera 3 & $x$ & $x$ & $x$ & $x$ & $\mathrm{Al}$ & $\mathrm{Al}$ & $x$ \\
\hline Pradiera 4 & $x$ & $x$ & $x$ & $x$ & $\mathrm{Al}$ & $\mathrm{Al}$ & $x$ \\
\hline Pradiera 5 & $x$ & $x$ & $x$ & $x$ & $\mathrm{Al}$ & $x$ & $x$ \\
\hline Pradiera 6 & $x$ & $x$ & $x$ & $x$ & $\mathrm{Al}$ & $x$ & $x$ \\
\hline Prati Grandi I & $x$ & $x$ & $x$ & $x$ & $x$ & $\mathrm{Al}$ & $x$ \\
\hline Prati Grandi 2 & $x$ & $x$ & $x$ & $x$ & $x$ & $\mathrm{Al}$ & $x$ \\
\hline Prati Grandi 3 & $x$ & $x$ & $x$ & $x$ & $x$ & $\mathrm{Al}$ & $x$ \\
\hline Prato Longo I & $x$ & $x$ & $x$ & $x$ & $\mathrm{Al}, \mathrm{Cg}$ & $\mathrm{Al}$ & $x$ \\
\hline Prato Longo 2 & $x$ & $x$ & $x$ & $x$ & $\mathrm{Al}^{\circ}$ & $\mathrm{Al}$ & $x$ \\
\hline Prato Longo 3 & $x$ & $x$ & $x$ & $x$ & $x$ & $\mathrm{Al}$ & $x$ \\
\hline Rivone I & $x$ & $x$ & $x$ & $x$ & $\mathrm{Cg}, \mathrm{Al}, \mathrm{Vy}$ & $\mathrm{Cg}, \mathrm{Al}, \mathrm{Vy}$ & $x$ \\
\hline Rivone 2 & $x$ & $x$ & $x$ & $x$ & $x$ & $\mathrm{Al}$ & $x$ \\
\hline
\end{tabular}


Table 2: Inventories (part B) (Continued)

\begin{tabular}{|c|c|c|c|c|c|c|c|}
\hline Rivone 3 & $x$ & $x$ & $x$ & $x$ & $x$ & $\mathrm{Al}, \mathrm{Vy}$ & $x$ \\
\hline S. Antonio 2 & $x$ & $x$ & $x$ & $x$ & $x$ & Og & W \\
\hline S. Antonio 3 & $x$ & $x$ & $x$ & $x$ & $x$ & $\mathrm{Og}$ & W \\
\hline Saggiano I & $x$ & $x$ & $x$ & $x$ & $\mathrm{Cg}$ & $x$ & w \\
\hline Saggiano 2 & $x$ & $x$ & $x$ & $x$ & $\mathrm{Al}, \mathrm{Cg}$ & $x$ & w \\
\hline Saggiano 3 & $x$ & $x$ & $x$ & $x$ & W & $x$ & W \\
\hline Saggiano 4 & $x$ & $x$ & $x$ & $x$ & $\mathrm{Cg}, \mathrm{W}, \mathrm{Al}$ & $x$ & W \\
\hline Saramazzo I & $x$ & $x$ & $x$ & $x$ & $\mathrm{Al}, \mathrm{Vy}$ & $\mathrm{Al}, \mathrm{Vy}$ & $\mathrm{Al}, \mathrm{Vy}$ \\
\hline Saramazzo 2 & $x$ & $x$ & $x$ & $x$ & $\mathrm{Al}, \mathrm{Og}$ & $\mathrm{Al}$ & $\mathrm{Al}$ \\
\hline Saramazzo 3 & $x$ & $x$ & $x$ & $x$ & $\mathrm{Al}, \mathrm{Vy}$ & $\mathrm{Al}, \mathrm{Vy}$ & $\mathrm{Al}, \mathrm{Vy}$ \\
\hline Saramazzo 4 & $x$ & $x$ & $x$ & $x$ & $\mathrm{Al}, \mathrm{Vy}$ & $\mathrm{Al}, \mathrm{Vy}$ & $\mathrm{Al}, \mathrm{Vy}$ \\
\hline Saramazzo 5 & $x$ & $x$ & $x$ & $x$ & $\mathrm{Al}, \mathrm{Vy}$ & $\mathrm{Al}$ & $\mathrm{Al}$ \\
\hline Saramazzo 6 & $x$ & $x$ & $x$ & $x$ & $x$ & $\mathrm{Al}$ & $\mathrm{Al}$ \\
\hline Saramazzo 7 & $x$ & $x$ & $x$ & $x$ & $x$ & $\mathrm{Al}, \mathrm{Vy}, \mathrm{Cg}$ & W \\
\hline Saramazzo 8 & $x$ & $x$ & $x$ & $x$ & $x$ & $\mathrm{Al}, \mathrm{Vy}$ & $A l, V_{y}$ \\
\hline Saramazzo 9 & $x$ & $x$ & $x$ & $x$ & $x$ & $\mathrm{Al}, \mathrm{Vy}$ & $\mathrm{Al}, \mathrm{Vy}$ \\
\hline Scandolara $2 *$ & $x$ & $x$ & $x$ & $x$ & $x$ & $\mathrm{Cg}, \mathrm{W}$ & w \\
\hline Scandolara $3 *$ & $\hat{x}$ & $\hat{x}$ & $\hat{x}$ & $\hat{x}$ & $\hat{x}$ & W & w \\
\hline Scandolara $4^{*}$ & $x$ & $x$ & $x$ & $x$ & $x$ & $\mathrm{~W}, \mathrm{Cg}$ & W \\
\hline Scandolara $5^{*}$ & $x$ & $x$ & $x$ & $x$ & $x$ & $w^{\circ}$ & W \\
\hline Scoglio del Zer & $x$ & $x$ & $x$ & $x$ & Ar (walnut) & $x$ & $x$ \\
\hline Selva & $x$ & $x$ & $x$ & $x$ & W & $x$ & $x$ \\
\hline Serralunga & $x$ & $x$ & $x$ & $x$ & $x$ & W & $x$ \\
\hline Suasca & $x$ & $x$ & $x$ & $x$ & $x$ & $\mathrm{Cg}, \mathrm{W}$ & $x$ \\
\hline Taramasco I & $x$ & $x$ & $x$ & $x$ & $\mathrm{Cg}$ & $x$ & $x$ \\
\hline Taramasco 2 & $x$ & $x$ & $x$ & $x$ & $\mathrm{Al}$ & $\mathrm{x}$ & $x$ \\
\hline Termini & $x$ & $x$ & $x$ & $x$ & $\mathrm{Al}$ & $x$ & W \\
\hline Tra la Serra & $x$ & $x$ & $x$ & $x$ & $\mathrm{Al}, \mathrm{Vy}$ & $\mathrm{Al}, \mathrm{Vy}$ & $x$ \\
\hline Tra le coste I & $x$ & $x$ & $x$ & $x$ & $\mathrm{Vy}, \mathrm{Ar}, \mathrm{Al}$ & W & $x$ \\
\hline Tra le coste 10 & $x$ & $x$ & $x$ & $x$ & $x$ & $\mathrm{Al}, \mathrm{Cg}$ & $x$ \\
\hline Tra le coste II & $x$ & $x$ & $x$ & $x$ & $x$ & $\mathrm{Cg}, \mathrm{Al}$ & $x$ \\
\hline Tra le coste 2 & $\hat{x}$ & $\hat{x}$ & $\hat{x}$ & $x$ & $\widehat{A l}$ & $\mathrm{Al}$ & $\hat{x}$ \\
\hline Tra le coste 3 & $x$ & $x$ & $x$ & $x$ & $\mathrm{Vy}, \mathrm{Ar}, \mathrm{Al}$ & $\mathrm{Al}$ & $x$ \\
\hline Tra le coste 4 & $x$ & $x$ & $x$ & $x$ & $\mathrm{Al}$ & $\mathrm{Al}$ & $x$ \\
\hline Tra le coste 5 & $\hat{x}$ & $\hat{x}$ & $\hat{x}$ & $\hat{x}$ & $\mathrm{Cg}, \mathrm{Vy}$ & $\mathrm{Cg}$ & $\hat{x}$ \\
\hline Tra le coste 6 & $x$ & $x$ & $x$ & $x$ & $\mathrm{Cg}, \mathrm{W}$ & $\mathrm{Cg}$ & $x$ \\
\hline Tra le coste 7 & $x$ & $x$ & $x$ & $x$ & $\mathrm{Cg}$ & $\mathrm{Cg}$ & $x$ \\
\hline Tra le coste 8 & $x$ & $x$ & $x$ & $x$ & $\mathrm{Vy}, \mathrm{M}, \mathrm{Al}$ & $\mathrm{Vy}, \mathrm{Cg}$ & $x$ \\
\hline Tra le coste 9 & $x$ & $x$ & $x$ & $x$ & $x$ & $\mathrm{Al}^{\circ}$ & $x$ \\
\hline Tra le coste di sopra & $\hat{x}$ & $\hat{x}$ & $\hat{x}$ & $\hat{x}$ & $\hat{x}$ & $\mathrm{Cg}$ & $\hat{x}$ \\
\hline Tra le coste di sotto & $x$ & $x$ & $x$ & $x$ & $x$ & $\mathrm{Al}, \mathrm{Cg}$ & $x$ \\
\hline Tra le vigne & $x$ & $x$ & $x$ & $x$ & $x$ & $\mathrm{Cg}, \mathrm{W}$ & $x$ \\
\hline Trombassa & $x$ & $x$ & $x$ & $x$ & $x$ & $\mathrm{Cg}, \mathrm{W}$ & $x$ \\
\hline$V a^{\prime} 2$ & $x$ & $x$ & $x$ & $x$ & $\mathrm{Cg}, \mathrm{Og}$ & $\mathrm{Cg}, \mathrm{Og}$ & $x$ \\
\hline$V a^{\prime} 3$ & $x$ & $x$ & $x$ & $x$ & $\mathrm{Cg}$ & $\mathrm{Cg}, \mathrm{W}$ & $\hat{x}$ \\
\hline$V a^{\prime} 4$ & $x$ & $x$ & $x$ & $x$ & $\mathrm{Og}$ & Og & $x$ \\
\hline$V a^{\prime} 5$ & $x$ & $x$ & $x$ & $x$ & $\mathrm{Al}$ & W & $x$ \\
\hline$V a^{\prime} 6$ & $x$ & $x$ & $x$ & $x$ & $\mathrm{Cg}, \mathrm{Og}$ & w & $x$ \\
\hline$V a^{\prime} 7$ & $x$ & $x$ & $x$ & $x$ & $\mathrm{Vy}, \mathrm{W}$ & w & $x$ \\
\hline Va' 8 & $\hat{x}$ & $\hat{x}$ & $\hat{x}$ & $\hat{x}$ & $\mathrm{Al}, \mathrm{Og}$ & Og & $\hat{x}$ \\
\hline$V a^{\prime} 9$ & $x$ & $x$ & $x$ & $x$ & $\mathrm{Og}$ & $\mathrm{Og}$ & $x$ \\
\hline$V a^{\prime} 10$ & $x$ & $x$ & $x$ & $x$ & $\mathrm{Vy}, \mathrm{Ar}, \mathrm{Al}$ & $x$ & $x$ \\
\hline$V a^{\prime} / I$ & $x$ & $x$ & $x$ & $x$ & $\mathrm{Al}, \mathrm{Og}$ & $\mathrm{Al}, \mathrm{Og}$ & $x$ \\
\hline Val del Gallo I & $\hat{x}$ & $\hat{x}$ & $\hat{x}$ & $x$ & $\mathrm{Vy}, \mathrm{Ar}, \mathrm{Al}$ & $x$ & $\hat{x}$ \\
\hline Val del Gallo 2 & $\hat{x}$ & $\hat{x}$ & $\hat{x}$ & $x$ & $\mathrm{Vy}, \mathrm{Ar}, \mathrm{Al}$ & $\hat{x}$ & $\hat{x}$ \\
\hline Val del Gallo 3 & $x$ & $x$ & $x$ & $x$ & $\mathrm{Vy}, \mathrm{Ar}, \mathrm{Al}$ & $\mathrm{x}$ & $x$ \\
\hline Val del Gallo 4 & $\hat{x}$ & $\hat{x}$ & $\hat{x}$ & $\hat{x}$ & $\mathrm{Al}$ & $\hat{x}$ & $\hat{x}$ \\
\hline Val del Rio & $x$ & $x$ & $x$ & $x$ & $\mathrm{Al}, \mathrm{W}$ & $x$ & $x$ \\
\hline Val di Franeta I & $x$ & $x$ & $x$ & $x$ & W & w & W \\
\hline Val di Franeta 2 & $\hat{x}$ & $\hat{x}$ & $\hat{x}$ & $\hat{x}$ & $\mathrm{x}$ & W & W \\
\hline Val di Becco I & $\hat{x}$ & $\hat{x}$ & $\hat{x}$ & $x$ & $\mathrm{Cg}, \mathrm{W}$ & $x$ & $x$ \\
\hline Val di Becco 2 & $\hat{x}$ & $\hat{x}$ & $\hat{x}$ & $x$ & W & $x$ & $x$ \\
\hline Valdelloro I & $x$ & $x$ & $x$ & $x$ & $\mathrm{Cg}$ & $\mathrm{x}$ & W, Cg \\
\hline
\end{tabular}


Table 2: Inventories (part B) (Continued)

\begin{tabular}{|c|c|c|c|c|c|c|c|}
\hline Valdelloro 2 & $x$ & $x$ & $x$ & $x$ & $\mathrm{~W}$ & W & W \\
\hline Valdelloro 3 & $x$ & $x$ & $x$ & $x$ & W & W & W \\
\hline Valdelloro 4 & $x$ & $x$ & $x$ & $x$ & W & $x$ & W \\
\hline Valdelloro 5 & $x$ & $x$ & $x$ & $x$ & W, Cg & W, Cg & W \\
\hline Valdelloro 6 & $x$ & $x$ & $x$ & $x$ & $\mathrm{~W}, \mathrm{Cg}$ & $\mathrm{W}, \mathrm{Cg}$ & W \\
\hline Valdelloro 7 & $x$ & $x$ & $x$ & $x$ & $\mathrm{Cg}, \mathrm{W}$ & W & W \\
\hline Valetti & $x$ & $x$ & $x$ & $x$ & $x$ & W & W \\
\hline Valle & $x$ & $x$ & $x$ & $x$ & $x$ & Al & $x$ \\
\hline Valle della Vecchia & $x$ & $x$ & $x$ & $x$ & $\mathrm{Cg}$ & $\mathrm{Cg}, \mathrm{W}$ & W \\
\hline Valle di Vallone & $x$ & $x$ & $x$ & $x$ & $x$ & $\mathrm{Cg}, \mathrm{W}$ & $x$ \\
\hline Valle Grande I & $x$ & $x$ & $x$ & $x$ & $x$ & W & $x$ \\
\hline Valle Grande 2 & $x$ & $x$ & $x$ & $x$ & $x$ & w & $x$ \\
\hline Vanedi I & $x$ & $x$ & $x$ & $x$ & $\mathrm{Al}, \mathrm{Vy}$ & $M$ & $x$ \\
\hline Vanedi 2 & $x$ & $x$ & $x$ & $x$ & $x$ & $\mathrm{Al}$ & $x$ \\
\hline Vanzola & $x$ & $x$ & $x$ & $x$ & $x$ & w & $x$ \\
\hline Varcava I & $x$ & $x$ & $x$ & $x$ & $\mathrm{Cg}$ & $x$ & W \\
\hline Varcava 2 & $x$ & $x$ & $x$ & $x$ & w & $x$ & $W$ \\
\hline
\end{tabular}

Inventories, ordered for every toponym, of the Municipality of Pignone, for the years 1878, 1933 and recent times.

\section{Results}

In the "Niccolo V" Lunense Episcopal Archive of Sarzana, four inventories related to the Parish of St. Mary in Pignone were found and transcribed (Figure 3). The first archival documents date back to 1588; the next date back to 1663,1763 and 1828 [12-15].

\section{Year 1588}

Fields for forage production (meadow) and chestnut grove prevail (Tab. 1, Figure 3). Other crop types are scarce. The relationship between the number of fields (38) and the number of crop portions (CU: $38 / 46$ ) is 0.83 .

\section{Year 1663}

Chestnut groves prevail, with a small decrease in the total number of fields from 16 (in 1588) to 12. In the XVII century, olive cultivation, not mentioned in the previous inventory, seems to have become extensive. Hemp cultivation increases (from 1 to 8 fields). Three crops cultivated on the vine are present. The CU indicator is $1(33 / 33)$, as all inventoried fields are utilized for only one crop type.

\section{Year 1763}

Chestnut groves prevail in this period as well. A fair number of fields with mixed cultures are present with

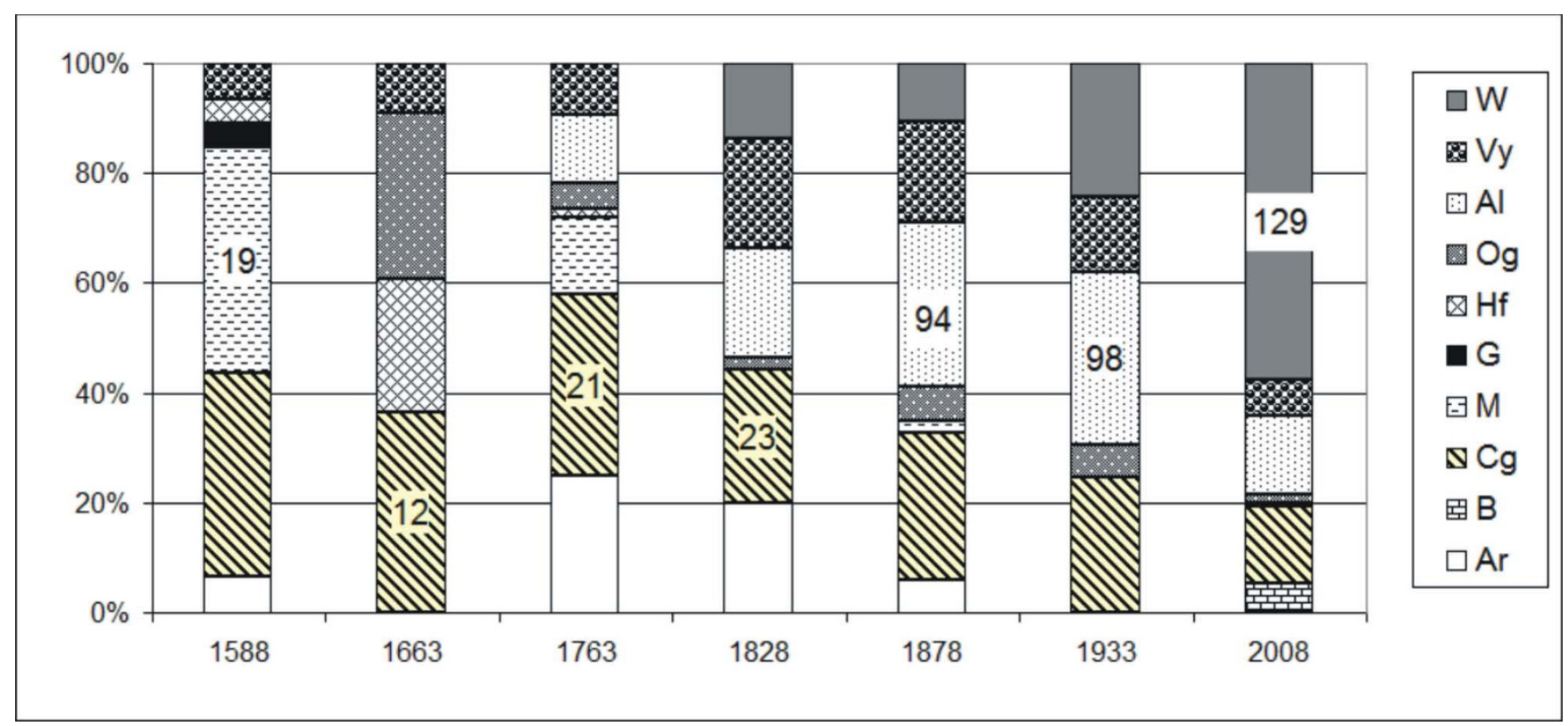

Figure 2

Frequency crop types. Percent frequency of crop types calculated for all toponyms. 
chestnut groves, arboretums, vineyards and arable lands. There is a decrease in areas planted exclusively with olive crops. The CU indicator is $0.56(36 / 64)$.

\section{Year 1828}

Mixed cultures with vine, arboretum and arable land prevailing. There was a substantial decrease in fields with exclusive chestnut production, but increased mixed areas with chestnut groves and woods. The CU indicator is 0.43 (41/95), the lowest value among the inventories studied.

In the State Archive of La Spezia, two inventories related to the Municipality of Pignone were found and transcribed. The archival documents date back to 1878 and $1933[16,17]$.

\section{Year 1878}

Arable land seems to be the main kind of crop, along with chestnut groves with an increase with respect to the previous (ecclesiastic) inventory. Woodlands are almost stable. On the other hand, the relationship between the number of fields and the number of crop (CU) portions increased to $0.61(194 / 316)$.

\section{Year 1933}

Arable land prevail. There was a substantial decrease in fields with chestnut groove and a increase of woods. The CU indicator is $0.73(226 / 311)$.

\section{Current}

To monitor the present landscape and crops, field surveys were carried out with the help of aerial photographs and the cadastral map of the Pignone territory. This allowed us

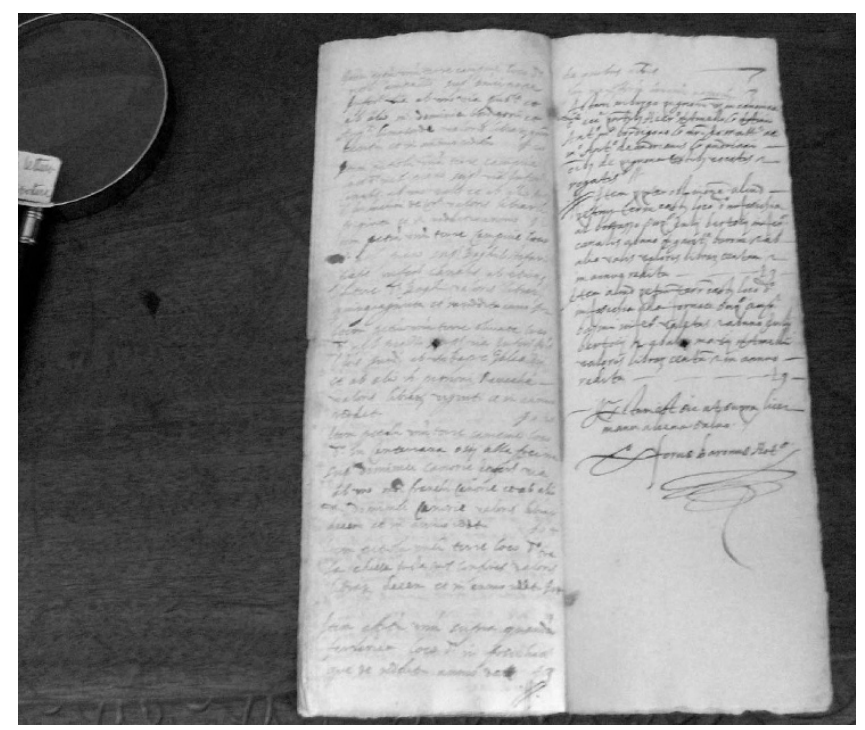

Figure 3

Ancient document. An ancient inventory of the Parish of St. Mary in Pignone conserved in the Archivi Vescovili Lunensi of Sarzana. to compare present and past situations linked to specific toponyms. The majority of ancient fields with chestnut groves and olive groves have been abandoned and partially reforested with cluster-pine woodland or mixed coppiced woodland (oaks, chestnuts, European hophornbeam, etc.). Some areas now have buildings.

At the other sites in Pignone, not linked to the toponyms noted in the inventories, a general reforestation has been surveyed. Vineyards and olive groves at present have been on the whole abandoned. The few arable lands consist of maize or vegetables. The CU indicator is $0.75(168 / 224)$.

\section{Discussion}

Ecclesiastic institutions were among the main landowners in Europe until the end of the XIX century. For this reason, such inventory data gives us qualitative and semiquantitative information regarding the types of crop change throughout time in an area of eastern Liguria. After the XIX century, data from the inventories of municipalities and from forest inventories are available for the same territory. Such information is in agreement with historical knowledge related to the study area and to other Mediterranean hill/mountain areas.

The chestnut, in historical times, was spread by Romans as plant for food. However, it is well documented that starting in the Middle Ages chestnut cultivation spread throughout the Mediterranean hill/mountain areas of Italy (then in Val di Vara), replacing part of the holm-oak woods [21-23]. In Val di Vara, domestication of the woodland chestnut tree spread during the XIV century, along with a strong increase in population [24]. Our data confirm such assertions and show that the main subsistence means in the study area from the XVI century to the first half of the XIX century was chestnut, and secondarily meadow in 1588, hempfield and olive in 1663, arboretum in 1763 and vine in 1828. In the study area in 1878 and 1933, arable land prevailed along with chestnut groves. High values of the CU indicator have evidenced few diversified crops in the XVI and XVII centuries. Crop diversification increased until the first half of the XIX century (low values of the CU indicator), then it decreased again (high values of the CU indicator).

In addition, in other Mediterranean regions, chestnut has been a tree historically cultivated and utilized as food, but also for a variety of other practices $[23,25]$. For example, chestnut could be utilized around crops in lines together with other trees as a barrier to wind, to stabilize soil and to support the harvest of leaves and branches for livestock [26]. In vineyards, vines were generally sustained by chestnut poles arranged in rows or pergolas [26].

In the study area and during some periods, a lesser part was played by olives and vines in agricultural activities. On the contrary, in the warmer areas of the Mediterranean 
basin close to the coast, olive groves and vineyards were the main crop types [5].

General investigation of the Ligurian Republic dated back to 1799 [27] and supplied data regarding forest vegetation and crop types in Val di Vara (some locations). For example, in the nearby Val di Pino, holm-oak and chestnut crops were present. In other locations in Val di Vara (San Pietro Vara and Varese Ligure) fields of chestnut, turkey-oak (Quercus cerris L.), downy-oak, European hop-hornbeam, beech (Fagus sylvatica L.), willow trees (Salix spp.) and olive trees were noted $[27,28]$. Crops with major production indices included chestnut, followed by wine, olive oil, wheat, rye, fruits, figs, legumes, potatoes (Solanum tuberosum L.), and vegetables $[18,19]$. Particularly interesting is the earlier presence of cultivated potatoes in Val di Vara. In Italy, the potato was introduced in Genova around 1585 by Carmelite Friars from Spain and Portugal [29]. However, the potato had begun to spread throughout all of Europe, and in eastern Liguria starting from the second half of the XVIII century $[30,31]$. Around Genova to the Val di Vara territory, the variety "Quarantina bianca" was cultivated [29]. The progressive increase in the cultivation and utilisation of potato and maize is considered one of the causes of the decrease in nutritional importance of the chestnut tree starting from the second half of the XIX century [32]. Our data support this assertion, as in the inventories of 1878 and 1933 arable land (Al) were the main crop type.

Starting from the XIX century (inventory of 1828), there was an increase of woodlands. This was very probably due to a transformation in the socio-economic conditions and also to the production of coal inside charcoal pits $[22,33]$. This practice disappeared everywhere after the Second World War, but gaps produced by the old charcoal spaces persist and are still visible in the woods [26]. In eastern Liguria (also in the Pignone territory), there has been increased extractive activities in this century in several modest mines for industrial, energy or building purposes, such as pyrite, kaolin, lignite, and sandstone. Such activity locally favoured the exploitation of the woods in the areas close to the mines [34].

Since the first half of the XIX century, chestnut ink disease, caused by Phytophthora cambivora and P. cinnamomi has become widespread in Europe [35]. In Italy and particularly in eastern Liguria, ink disease and chestnut disease (cancer of the bark) gravely damaged chestnut groves starting from the end of the XIX century until about 1950 [36-38].

Before the XX century, the agricultural system in the study area mainly produced food for self-sustaining local communities, parishes and landowners, and only occasionally for external commerce [26].

During the XX century, chestnut groves in the study area significantly decreased in importance. Such trends have been evidenced in upper Val di Vara [24]: in 1938, the chestnut woods (coppiced and trees) covered about $60 \%$ of the territory. After the Second World War, in Val di Vara there were strongly augmented plantations of the most degraded woodland (erosion) with coniferous trees [39]. In 1993, most of the territory was forested by mixed woodlands, turkey-oak woodlands and plantations (Pinus spp.), and only $15 \%$ of the area was occupied by chestnut coppice [24].

The recent forest census of the Liguria Region revealed that only about $22 \%$ of the forest surface of the whole Province of La Spezia is occupied by chestnut (comprising coppice); mixed coppiced woodland and cluster pine woodland represent $21.8 \%$ and $15.2 \%$ respectively [40]. The same forest census showed that in the Pignone territory, chestnut coppice represents 20\%, mixed woodland $27.60 \%$ (oaks, chestnuts, European hop-hornbeam, etc.), cluster pine woodland $13.1 \%$, olive grove $2.5 \%$, and other cultivated lands $9.7 \%$ [40].

In this context, the traditional elements of the rural landscape, in terms of chestnut groves and olive groves (terraces, stonewalls, etc.), have been not preserved, but rather have deteriorated or been destroyed [5]. We can assert that agriculture abandonment and changing socioeconomic conditions over the last 50 years have favoured a strong increase in cluster-pine woodland or mixed coppiced woodland (oaks, chestnuts, European hop-hornbeam, etc.) and partially in evergreen sclerophyllous forest and shrubland at the expense of cultivated areas, especially those with chestnuts and olives [7]. A similar trend has been observed for coastal areas of eastern Liguria, where vineyard, the dominant crop type, has been mainly substituted by cluster pine woodland $[40,41]$

As evidenced in other Mediterranean hill/mountain areas, this has lead to a simplification of the landscape (an increase in the CU indicator) due to abandonment of agricultural practices and reforestation [42,31].

\section{Conclusion}

This study has examined crop/land cover changes during the last five centuries in an area of eastern Liguria (Italy) through examination of archival documents taken from ecclesiastic and municipal inventories, and current field surveys. In accordance with other studies conducted in the Mediterranean region, we show evidence of the traditional use of chestnut as one of the main source of food in the hill/mountain areas of eastern Liguria in the XVI, XVII and XVIII centuries. This work has highlighted how ancient documents, which are at present not generally used in ethnobotanical studies, can be very useful for enhancing the knowledge of agricultural and livestock practices and of subsistence methods favoured by local populations during a particular time. This study has also shown that inventories (but also other types of documents) are very useful for 
the historical reconstruction of the landscape by means of relative comparison of cultivated fields, anthropogenic woods and naturally reforested areas.

\section{Competing interests}

The authors declare that they have no competing interests.

\section{Authors' contributions}

RG and EG found and transcribed the ancient document. RG and SG wrote the paper and prepared the tables and figures. All authors read and approved the final manuscript.

\section{Acknowledgements}

This research was funded by Milano-Bicocca University. The authors are grateful to Enzo Freggia, director of the Biblioteca Niccolò V - Archivi Vescovili Lunensi of Sarzana, and to the archivists of the State Archive of La Spezia for assistance and technical support in this study.

\section{References}

I. Nabhan GP: Agrobiodiversity Change in a Saharan Desert Oasis, 1919-2006: Historic Shifts in Tasiwit (Berber) and Bedouin Crop Inventories of Siwa, Egypt. Economic Botany 2007, 6I:31-43.

2. Prosperi A: Il Concilio di Trento: una introduzione storica Torino: Einaudi; 2001 .

3. Moreno D, Cevasco R, Guido MA, Montanari C: L'approccio storico-archeologico alla copertura vegetale: il contributo dell'archeologia ambientale e dell'ecologia storica. In La biologia vegetale per i beni culturali, Vol II, Conoscenza e valorizzazione Edited by: Caneva G. Firenze, Nardini Editore; 2005:463-498.

4. Dahlström A: Grazing dynamics at different spatial and temporal scales: examples from the Swedish historical record A.D. 1620-1850. Vegetation History and Archaeobotany 2008, 17:563-572.

5. Kizos T, Kolouri M: Agricultural landscape dynamics in the Mediterranean: Lesvos (Greece) case study using evidence from the last three centuries. Environmental Science and Policy 2006, 9:330-342.

6. Poyatos R, Latron J, Llorens P: Land use and land cover change after agricultural abandonment: the case of a Mediterranean mountain area (Catalan pre-Pyrenees). Mountain Research and Development 2003, 23:362-368.

7. MacDonald D, Crabtree JR, Wiesinger G, Dax T, Stamou N, Fleury P Gutierrez Lazpita J, Gibon A: Agricultural abandonement in mountain areas of Europe: environmental consequences and policy response. Journal of Environmental Management 2000, 59:47-69.

8. van Etten J: Changes in farmers' knowledge of maize diversity in highland Guatemala, 1927/37-2004. Journal of Ethnobiology and Ethnomedicine 2006, 2:12.

9. Starnini E, Ottomano C, Nisbet R: Risultati di un intervento di emergenza al Castellaro di Pignone (SP). Rivista di Archeologia 1992, 16:50-64.

10. Calcagno D, (Ed): Pignone e le sue vie tra Medioevo ed Età Moderna Chiavari: Piemme grafica; $200 \mathrm{I}$

II. Rivas-Martìnez S, Penas A, Diaz TE: Biogeographic map of Europe. Cartographic Service. 2004 [http://www.globalbiocli matics.org/form/maps.htm]. Spain: University of León

12. Archivio Vescovile Lunense of Sarzana: Serie Parrocchiali $n^{\circ} / 5$, Inventario. Pignone I588.

13. Archivio Vescovile Lunense of Sarzana: Serie Parrocchiali $n^{\circ} / 5$, Inventario. Pignone 1663:1663.

14. Archivio Vescovile Lunense of Sarzana: Serie Parrocchiali $n^{\circ} / 5$ Inventario. Pignone 1763.

15. Archivio Vescovile Lunense of Sarzana: Serie Parrocchiali $n^{\circ} / 5$, Inventario. Pignone 1828.

16. Archivio di Stato La Spezia: Vecchio catasto terreni del Comune di Pignone, Partitario $n^{\circ} 3$, coll. 1048. Pignone 1878.

17. Archivio di Stato La Spezia: Vecchio catasto terreni del Comune di Pignone, Partitario $n^{\circ} 3$, coll. 1049. Pignone 1933.
18. Moreno D: Per una storia delle risorse ambientali. Pratiche agro-silvo-pastorali e coltura vegetale in alta Val di Vara. Quaderni storici 1988, 69:941-979.

19. Ceschi D: Noterelle sugli orti della Lunigiana storica. In Cronaca e storia di Val di Magra, anno XX-XXI Aulla: Centro Aullese di ricerche e studi lunigianesi; 200I:I09-I 20.

20. Conti F, Abbate G, Alessandrini A, Blasi C: An annotated checklist of the Italian vascular flora Roma: Palombi Editori; 2005.

21. Gabbrielli A: La civiltà del castagno. Monti e Boschi 1994, 45:3.

22. Cevasco R, Moreno D, Poggi G, Rackham O: Archeologia e storia della copertura vegetale: esempi dall'Alta Val di Vara. Memorie dell'Accademia Lunigianese di Scienze "Giovanni Capellini" I999, 69:24I-26I.

23. Conedera $M$, Krebs $P$, Tinner $W$, Pradella M, Torriani D: The cultivation of Castanea sativa (Mill.) in Europe, from its origin to its diffusion on a continental scale. Vegetation History and Archaeobotany 2004, 13:161-179.

24. Gabbrielli A: Appunti storici sul paesaggio forestale dell'Alta Val di Vara. Monti e Boschi 1997, 48:4-9.

25. Manuel Pardo-de-Santayana M, Tardío J, Blanco E, Carvalho AM, Lastra JJ, San Miguel E, Morales R: Traditional knowledge of wild edible plants used in the northwest of the Iberian Peninsula (Spain and Portugal): a comparative study. Journal of Ethnobiology and Ethnomedicine 2007, 3:27.

26. Farina $\mathrm{A}$ : The cultural landscape of Lunigiana. Memorie dell'Accademia Lunigianese di Scienze "Giovanni Capellini" 1996, 66:83-90

27. Faggioni PE: La Lunigiana nell'Inchiesta generale della Repubblica Ligure (1799). Memorie dell'Accademia Lunigianese di Scienze "Giovanni Capellini" 200 I, 7 I: I I-202.

28. Archivio di Stato La Spezia: Comune di Varese Ligure. Stato Generale de Boschi del Comune di Varese Ligure Varese Ligure; 1798.

29. Angelini M: Le patate della tradizione rurale sull'Appennino ligure Chiavari: Grafica Piemme; 2008.

30. Giacomini V, Fenaroli L: La Flora. Conosci I'Italia Volume II. Milano: Touring Club Italiano; 1958.

31. Hawkes JG, Francisco-Ortega J: The early history of the potato in Europe. Euphytica 1993, 70:1-7.

32. Conedera M, Stanga P, Lischer C, Stockli V: Competition and dynamics in abandoned chestnut orchards in southern Switzerland. Ecologia Mediterranea 2000, 26:101-II2.

33. Paola G, Nardelli R, Giacomazzi F, Simonini L, Degiorgis L, De Nevi P, (Eds): Fiori e piante alla Spezia e Lunigiana La Spezia: Cassa di Risparmio della Spezia S.p.A., Luna Editore, Società Editrice Ligure Apuana; 1998.

34. Del Soldato M, Pintus S, Zuffardi P: Le risorse estrattive della provincia della Spezia. Memorie dell'Accademia Lunigianese di Scienze "Giovanni Capellini" 1993, 62/63:47-I 22.

35. Vettraino AM, Natili G, Anselmi N, Vannini A: Recovery and pathogenicity of Phytophthora species associated with a resurgence of ink disease in Castanea sativa in Italy. Plant Pathology 200I, 50:90-96.

36. Gibelli G: Nuovi studi sulla malattia del Castagno detta dell'inchiostro. Memorie dell'Accademia delle Scienze dell'Istituto di Bologna I883, 4:287-3|4

37. Piccioli R: Pane d'albero e albero del pane. La coltura del castagno in Lunigiana. In Bagnone e il suo territorio Edited by: Bianchi I. Bagnone, Comune di Bagnone; 1990:289-295.

38. Heiniger $U$, Rigling D: Biological control of chestnut blight in Europe. Annual Review of Phytopathology 1994, 32:58I-599.

39. I.P.L.A. (Istituto per le Piante da Legno e I'Ambiente): I tipi forestali della Liguria Genova: Regione Liguria Dipartimento Agricoltura, protezione civile e Turismo, ERGA Edizioni; 2008.

40. S.P.I.R.L. (Servizio Prevenzione Incendi Regione Liguria): Istruzioni per il rilevamento dei popolamenti forestali in Regione Liguria 2000 [http:// www.agriligurianet.it/cgi-bin/liguria/agrinet3/ep/home.do]. Genova: Dipartimento Agricoltura, Parchi e Foreste Regione Liguria, Corpo Forestale

4I. Storti M: II paesaggio storico delle Cinque Terre. Individuazione di regole per azioni di progetto condivise. Quaderni della Ri-Vista Ricerche per la Progettazione del Paesaggio 2005, 2(I): I I3-I 24.

42. Agnolotti $M$ : The degradation of traditional landscape in a mountain area of Tuscany during the 19th and 20th centuries: implication for biodiversity and sustainable management. Forest Ecology and Management 2007, 249:5-17. 